\section{Globalização farmacêutica e cidadania biológica: notas sobre a implementação da profilaxia pós-exposição no Rio Grande do Sul, Brasil}

\author{
Pharmaceutical globalization and biological \\ citizenship: notes on the implementation of \\ post-exposure prophylaxis in the State \\ of Rio Grande do Sul, Brazil
}

\author{
Globalización farmacéutica y ciudadanía \\ biológica: notas sobre la implementación de la \\ profilaxis posexposición en Río Grande do Sul, \\ Brasil
}

\section{Resumo}

Com base em uma pesquisa etnográfica multissituada no campo da implementação da profilaxia pós-exposição (PEP) em serviços de saúde do Estado do Rio Grande do Sul, Brasil, este estudo discute aspectos da micropolítica de oferta das "novas tecnologias de prevenção" mediante conceitos de globalização farmacêutica e cidadania biológica. A prevenção combinada sugere 0 emprego conjunto de estratégias comportamentais, biomédicas e estruturais para enfrentar o HIV, porém, nas margens (co)construídas dessa politica, as ações dos serviços de saúde observados no Rio Grande do Sul evidenciaram a profunda fragmentação dessa combinação na rede, privilegiando-se o acesso à medicação. Estimulada por um cenário de globalização farmacêutica, a forma como a PEP é acessada e ofertada aos usuários enseja a articulação de uma cidadania biológica que implica o direito à prevenção, nesse caso por meio do direito individual de consumir a medicação. Contudo, o acesso a tal direito é perpassado por condicionamentos morais atrelados às categorias de risco e pelo contexto social dos usuários, reproduzindo-se iniquidades em saúde e empobrecendo a abordagem de aconselhamento. Discute-se a necessidade de que estratégias de prevenção combinada fortaleçam a abordagem das dimensões sociais e programáticas da epidemia, o que também potencializará a atenção às vulnerabilidades individuais na perspectiva da saúde integral.

HIV; Síndrome de Imunodeficiência Adquirida; Profilaxia Pós-exposição; Vulnerabilidade em Saúde
Adriano Henrique Caetano Costa 1

Tonantzin Ribeiro Gonçalves 2

doi: 10.1590/0102-311X00041420

\author{
Correspondência \\ T. R. Gonçalves \\ Rua Canísio Binsfeld 271, Porto Alegre, RS 91788-060, Brasil. \\ tonanrib@yahoo.com.br \\ 1 Universidade Federal do Rio Grande do Sul, Porto Alegre, \\ Brasil. \\ 2 Universidade do Vale do Rio dos Sinos, São Leopoldo, Brasil.
}




\section{Introdução}

A epidemia do HIV/aids é uma preocupação para a saúde pública brasileira e, em especial, para o Estado do Rio Grande do Sul que detêm altas taxas de incidência de aids, de infecção pelo HIV e de mortalidade por aids há muitos anos 1,2. Mais de 50\% dos casos de HIV no Rio Grande do Sul concentram-se na Região Metropolitana de Porto Alegre 2. Recentemente, esforços foram empreendidos visando ao desenvolvimento de uma política regionalizada para a epidemia. Por exemplo, a Cooperação Interfederativa, firmada entre o Ministério da Saúde, Secretaria de Estado da Saúde e 15 municípios prioritários, objetivou responder à situação epidemiológica das IST/aids e hepatites virais no Rio Grande do Sul pela construção de uma agenda organizada por prioridades e estratégias específicas. O acordo esteve vigente entre 2015 e 2017, e as ações incidiram sobre a mortalidade por aids, coinfecção por tuberculose (TB) e hepatites virais, prevenção para populações-chave (usuários de drogas, profissionais do sexo, homens que fazem sexo com homens - HSH, travestis e transexuais), aumento da capacidade e eficiência dos serviços, expansão da oportunidade de acesso ao diagnóstico rápido e aprimoramento da gestão.

Nesse contexto, desde 2012, a Política de Prevenção Combinada foi implementada no Rio Grande do Sul, primeiramente, com a testagem rápida na atenção básica e, depois, com a profilaxia pós-exposição (PEP) 3. No âmbito da Cooperação, a estratégia foi a ampliação e qualificação da oferta da PEP nos serviços da Rede de Urgência e Emergência (RUE), Serviço de Assistência Especializada (SAE) e Centro de Testagem e Aconselhamento (CTA) dos municípios prioritários.

Para além do cenário local, as propostas atuais da política de prevenção articulam-se a aspectos históricos da resposta à aids no mundo e no Brasil ${ }^{4}$. Inicialmente, partiu-se da noção de grupos de risco, que associava a doença a alguns subgrupos populacionais já discriminados e facilitava ações limitadas de prevenção, mantendo a aids como um problema dos "outros" 5. Com a descoberta do agente causador, um novo discurso sobre o risco de infecção pelo HIV emergiu, atrelando-o a comportamentos individuais que seriam o alvo das estratégias preventivas, contribuindo para estender algumas ações para a população em geral. Posteriormente, com a contribuição dos movimentos sociais e do ativismo pelos direitos humanos, liderados por homossexuais e pessoas vivendo com HIV/aids (PVHA) surge o conceito de "vulnerabilidade" procurando compreender as suscetibilidades diferenciais ao vírus entre os grupos populacionais e que (re)produziam iniquidades sociais 5 . A vulnerabilidade abarca as relações complexas entre os planos individual, social e programático 6, e a prevenção ao HIV/aids ganha novos sentidos, com estratégias sobre os determinantes estruturais da epidemia como estigma, discriminação e normas de gênero.

O momento atual dos modelos de prevenção do HIV se caracteriza pela fusão entre prevenção e tratamento, com a introdução de estratégias de prevenção baseadas no uso de antirretrovirais (ARV) 4. Investimentos econômicos foram canalizados para pesquisa biomédica sobre a biologia da infecção precoce e a ação dos ARV para prevenção. O reconhecimento da eficácia dessas intervenções mudou o paradigma, surgindo o conceito de prevenção combinada do HIV, em 2009, nos documentos oficiais norte-americanos de resposta ao HIV/aids, como tipo ideal para a prevenção. Assim, as abordagens preventivas passaram a se basear no uso combinado da PEP, da profilaxia pré-exposição (PrEP) e do tratamento como prevenção (TASP) somados às estratégias comportamentais e estruturais consagradas e entendendo que elas se reforçariam mutuamente ${ }^{4}$. Com o fortalecimento recente do uso da PEP, PrEP e TASP, a prevenção combinada compõe o marco teórico da política de HIV/aids no Brasil 4.

Em particular, a PEP estava disponível no Sistema Único de Saúde (SUS) desde 1999, objetivando ampliar formas de prevenção de infecções em situações de acidentes com material biológico, violência sexual ou exposição sexual consentida com pessoa soropositiva ou de status sorológico desconhecido em que houve penetração sem uso de preservativos com sua ruptura ou deslizamento 7 . Porém, sua aplicação restringia-se aos casos de estupro e acidentes ocupacionais. Em 2017, o esquema ARV da PEP para HIV foi simplificado, sugerindo que a avaliação do risco privilegiasse a situação de exposição do usuário e não mais sua categoria de exposição (acidente com material biológico, violência sexual ou exposição sexual consentida). Tal mudança compõe a estratégia de ampliação do uso da PEP, o que gerou um aumento da sua oferta, conforme dados do Ministério da Saúde (http://indicadores.aids. gov.br, acessado em Mar/2020). 
O uso de ARV como estratégia preventiva detém potencial de atingir populações em contextos de maior vulnerabilidade, como os HSH, usuários de drogas e profissionais do sexo, sendo uma alternativa eficaz frente às dificuldades de manter o uso consistente de preservativos. Porém, debates têm surgido quanto à excessiva ênfase no uso de medicamentos em detrimento da real combinação com ações de cunho programático 8,9. Nesse sentido, o presente artigo objetiva problematizar os desafios concernentes à oferta da PEP no contexto epidemiológico do Rio Grande do Sul, partindo do cenário macropolítico de aids no Brasil e dos conceitos de globalização farmacêutica 10 e cidadania biológica 11 . Com um olhar interseccional se pretende explorar os contextos diferenciais de acesso e oferta à PEP.

Ao se falar de cidadania, entende-se que o conceito foi elaborado com base em conflitos históricos que caracterizaram a tensão entre exclusão e inclusão na vida social, política e econômica. Sobre isso, Hannah Arendt 12 discute a perspectiva ampla de cidadania, que se refere ao pertencimento à humanidade, e cidadania ativa que, resumidamente, indica o pertencimento a uma comunidade política organizada. Ainda, o conceito de "quem" descreve o reconhecimento da alteridade dos sujeitos, que fundamenta a política. No âmbito da prevenção ao HIV, problematizou-se como essas perspectivas de cidadania parecem ser enlanguescidas entre populações vulnerabilizadas que têm, de modo geral, frágil acesso a direitos sociais básicos, recuperando, pelo uso da PEP, uma cidadania restrita à dimensão biológica.

Nessa direção, o regime de globalização farmacêutica contemporâneo instaura uma lógica medicalizante de consumo em que se busca garantir os direitos individuais, e os indivíduos deixam de ser cidadãos para serem consumidores. Tal mudança no papel do sujeito social segue na contramão do debate da cidadania e do sujeito como um ser político. Diante disso, buscar-se-á discutir como a oferta e acesso à PEP se coloca em um cenário de restrição tanto de recursos públicos para a saúde quanto nas estratégias estruturais de combate ao HIV 13,14.

Desse modo, esta pesquisa buscou fornecer à gestão pública de saúde evidências para a avaliação da implementação da PEP, indicando possíveis lacunas para os profissionais dos serviços e, no possível, sugestões para sua melhoria. Entende-se que os processos de trabalho em saúde se inserem num contexto conjectural mais amplo que pode afetar a execução de tarefas no cotidiano dos serviços de saúde, sem que seja possível culpabilizar individualmente os profissionais por determinadas decisões.

\section{Percurso metodológico}

A pesquisa etnográfica que subsidia as reflexões a seguir se deu entre março de 2017 e junho de 2018, a partir de visitas, conversas informais, observações e entrevistas com 12 profissionais de saúde e usuários da PEP, na Região Metropolitana de Porto Alegre. Entende-se a etnografia não como uma simples técnica de coletar dados, mas como forma de apreender e interpretar a realidade, caracterizando-se pela presença física e de longa duração do pesquisador em campo na busca por desvelar aspectos não aparentes 15 .

O trabalho de campo foi conduzido pelo primeiro autor que também era consultor técnico da política de HIV do Rio Grande do Sul, responsável pelas ações de prevenção combinada e articulação com a sociedade civil. Essa inserção possibilitou uma perspectiva privilegiada da política e das estratégias de implementação das ações de prevenção combinada no âmbito estadual. Portanto, ao enfocar as categorias e classificações locais, os pesquisadores procuram abandonar o que é descrito, na antropologia, como uma "perspectiva de fora", para adotar aquilo que é descrito como uma "perspectiva de dentro" 16 . Geertz 16, por exemplo, propõe como procedimento metodológico os conceitos de "experiência distante" e o de "experiência próxima". A experiência do pesquisador é diferente da de outros indivíduos, pois ele se propõe a conhecer certa situação guiado por referências teóricas densamente constituídas. O pesquisador no campo encontra indivíduos/informantes inseridos em outro contexto, o que pode produzir uma "fusão de horizontes", nesse caso, entre a construção teórica do pesquisador - experiência distante - e o significado das ações e vivência dos nativos - experiência próxima 16. Entre "proximidade" e "distanciamento", os dados produzidos foram analisados por tensionamentos do campo e do diálogo com os conhecimentos teóricos acumulados sobre as políticas públicas de saúde e HIV/aids, como a categoria analítica desenvolvida procurou demonstrar. 
Para investigar a implementação e acesso à PEP pelos profissionais de saúde no Rio Grande do Sul, buscou-se contemplar, simultaneamente, a perspectiva de três atores sociais: o Departamento de IST/ AIDS e Hepatites Virais; a Coordenação Estadual de IST/AIDS do Rio Grande do Sul e municípios. Ao enfocar a articulação complexa entre aspectos difíceis de situar como a epidemia de aids, as políticas e ações de agências governamentais em esferas nacionais, estaduais e municipais, seguiu-se o raciocínio de Marcus 17 para o desenvolvimento de um trabalho de campo em sítios múltiplos que busca cotejar o objeto pelas diversas relações que se dão entre os interlocutores da pesquisa.

Fazer pesquisa em diferentes espaços implicou a construção de um corpus empírico heterogêneo e a discussão da relação entre esses múltiplos sítios e os dados que lhe foram constitutivos. Para acompanhar a implementação da PEP nos municípios prioritários foi preciso usar métodos e modos específicos de participação e observação em cada local, em graus variados de profundidade. A etnografia compreendeu inserções em três SAE de três cidades e em dois serviços da RUE, referências para dispensação da PEP em uma das cidades. Acompanhou-se a rotina de atendimento desses locais, com especial interesse na PEP, privilegiando as interações e significados atribuídos entre usuários, profissionais, gestores locais e estaduais. O presente relato privilegiará as reflexões produzidas pelo contato com um dos SAE situados na Região Metropolitana de Porto Alegre, que tinha um fluxo bem consolidado da PEP e no qual o pesquisador realizou 22 visitas de três a quatro horas de duração. Documentos oficiais do governo e publicações acadêmicas auxiliaram no diálogo e análise da experiência de campo.

A pesquisa foi aprovada pelo Comitê de Ética em Pesquisa da Universidade do Vale do Rio dos Sinos (processo no 1.868.853). Os participantes assinaram um Termo de Consentimento Livre e Esclarecido e as observações ocorreram após assentimento da coordenadora do serviço.

\section{PEP, cidadania biológica e biopolíticas de uma prevenção (des)combinada}

Nos vários locais e contatos ao longo do trabalho de campo, a dispensação da medicação da PEP era uma prioridade para os profissionais e serviços de saúde, como recomenda o protocolo clínico em que "ressalta-se que a ausência de médico infectologista não deve atrasar a prescrição da profilaxia" 7 (p. 32). Nesses casos, o documento recomenda que o usuário exposto inicie imediatamente a PEP e seja reavaliado o mais brevemente possível em um serviço de referência para eventual adequação do esquema 7. Como uma das profissionais afirmou, a medicação possui, na ótica estatal, grande peso no tratamento da PEP e, consequentemente, em toda estratégia de prevenção combinada: "Na verdade eu tenho aqui meu kitzinho, eu dou a medicação, tem toda a questão 'e a tua formação permite?'. Eu penso que, assim, eu foco a importância que é pro paciente, se não tem médico aqui, às vezes não tem, ele tem que ir pra um 24 horas e de repente pode deixar de fazer, às vezes o tempo da PEP já tá quase caducando porque não percebeu que estava chegando a 72 horas, então eu primo por isso, dar a medicação, sei que pode ter algum problema, vai que dê alguma alergia em alguém, mas eu até pergunto algumas coisas, mas prefiro dar medicação, essa é minha prioridade" (Assistente Social).

Os medicamentos se tornaram elementos-chave da política pública, excedendo uma forma de controle de cima para baixo, pois a oferta dessas tecnologias agencia a prevenção do HIV nos indivíduos para manutenção da vida. Os serviços de saúde e profissionais estão intimamente envolvidos nessa articulação biopolítica que busca governar populações e gerenciar corpos indóceis ao uso do preservativo. O jogo é garantir o acesso ao medicamento para os indivíduos, enquanto o cenário para uma efetiva combinação enfrentando os determinantes estruturais da infecção pelo HIV se mostra muito frágil. Por exemplo, na recepção do serviço havia uma cesta com vários preservativos masculinos, não estando disponíveis o preservativo feminino, o gel lubrificante ou informativos. As recepcionistas não realizavam qualquer acolhimento ou orientação mínima dos usuários, apenas encaminhando-os para a psicóloga, o enfermeiro e a assistente social, que realizavam, efetivamente, todo o atendimento. Ainda, questionados sobre a articulação intersetorial, a única ação destacada foi a participação no Programa Saúde na Escola, que se restringia às palestras ofertadas pelos profissionais do SAE às escolas participantes.

Num contexto de restrições de recursos, falta de formação e incentivo da gestão para articular ações e políticas sociais mais amplas de prevenção seja para a população em geral ou para subgrupos, 
era como se os profissionais pudessem se dirigir apenas para a garantia da não infecção dos indivíduos. Tais ações se desdobram num cenário em que o Estado é farmaceuticamente presente (via mercados), mas, em geral, institucionalmente ausente. Enquanto a bem-sucedida universalização do acesso aos ARV no Brasil como estratégia central de combate à epidemia de HIV se pautou, na década de 1990, no confronto com a indústria farmacêutica, é interessante observar como, paulatinamente, esse mercado se apropriou do discurso dos direitos humanos (na perspectiva restrita do consumo individual) para manter seus lucros, e a incorporação do acesso à PEP se coloca como uma possibilidade de expansão.

Assim, na lógica "pragmática" da globalização, os cidadãos são tratados como consumidores e têm interesses ao invés de necessidades. A governança neoliberal tomou essa nova forma como o principal paradigma da saúde pública, passando da prevenção para o acesso ao tratamento e, no terceiro momento, a incorporação do tratamento na prevenção. Dessa forma, entende-se que os direitos políticos vinculados à cidadania modificaram-se para a ideia de direitos biologicamente baseados 10 . Para Biehl et al. 18, estariam ainda adotando abordagens de "bala mágica", em que a entrega de tecnologia biomédica, independentemente da infraestrutura dos serviços e da qualificação profissional, é a norma, e as empresas farmacêuticas e os profissionais reproduzem o discurso de que o acesso aos medicamentos, por si só, é uma questão de direitos humanos.

Tais processos de cidadania representam, para Rose \& Novas ${ }^{11}$, o conjunto de políticas e práticas empregadas pelo Estado, que determinam quais indivíduos são cidadãos em potencial. Para esses autores, definições biológicas como as de raça, linhas de sangue, descendência e inteligência, inspiraram vários projetos de cidadania, relacionando as "concepções de cidadãos às crenças sobre a existência biológica dos seres humanos como indivíduos, como família e linhagem, como comunidades, como população e raças, e como espécie" 11 (p. 2). Para eles, as conformações em torno da cidadania biológica se transformam e se re-territorializam pelas injunções nacionais, locais e transnacionais da sociedade. Portanto, a PEP pode ser vista como uma estratégia de incorporação progressiva de conhecimento dominado por discursos e materialidades sobre HIV e aids, produzindo um discurso limitado sobre a cidadania em um contexto de grandes desigualdades como no Brasil.

Pode-se dizer que a genética abriu espaço para a criação de novas identidades, e "o laboratório se tornou uma espécie de fábrica para a criação de novas formas de vida" 19 (p. 13). Nesse sentido, assistimos à construção de identidades sociais/sexuais a partir da epidemia, como exemplo, pessoas que vivem com HIV/aids, casais sorodiscordantes, pessoa indetectável, dentre outros. Portanto, práticas clínicas e tecnologias biomédicas relacionadas ao HIV/aids (testes, ARV, contagem de células TCD4, genotipagem etc.) seriam formas de (bio)definição e (bio)identificação que incidem nos processos sociais e culturais relacionados à epidemia. Por exemplo, os profissionais acessados relataram que alguns usuários utilizavam a PEP mais de três vezes por ano, e que essas pessoas tinham banalizado a PEP, não temiam se infectar e, por isso, não utilizavam preservativos. $\mathrm{O}$ mesmo processo ocorreu com doenças como a sífilis nos anos 1920 e 1930 20, mas também quanto às doenças genéticas 21,22. Pode-se pensar que a oferta e o uso da PEP estejam agenciando modos particulares de gerenciamento do risco pelos indivíduos, implicando novas definições de si, num contexto de restrições de acesso a outros direitos básicos.

No horizonte da prevenção combinada, as dimensões individual, social e programática da vulnerabilidade ao HIV não se conectavam ao cotidiano de ações do serviço. A ênfase no acesso à medicação colocava as questões sociais e programáticas locais em segundo e terceiro planos da prevenção. Por exemplo, os usuários não chegavam ao serviço procurando pela PEP e muitos desconheciam a existência desse recurso ou tinham vergonha de solicitá-lo. Entretanto, pediam o Teste Rápido (TR) e, durante o aconselhamento, o profissional fazia a indicação da PEP: "O paciente não diz o que é mas quer conversar com alguém ou então veio fazer o teste. Ai eu chamo e pergunto pra pessoa o que ela veio fazer, normalmente vem em função de um teste ou porque rompeu o preservativo, aí eu pergunto quanto tempo faz e se teve a indicação da PEP. Eu já apresento a PEP, o que significa a PEP, o que dentro desse prazo ele ainda pode tomar uma medicação e ai apresento como é que funciona a PEP" (Psicóloga).

"Quando identificamos exatamente a que ele veio, ele dá um sinal que é pra PEP ou ele traz um discurso indicando a PEP. As meninas da portaria já encaminham para nós um fluxo, o documento padrão pra registro da PEP. Quando elas não conseguem identificar entra como um teste rápido" (Enfermeiro).

Percebe-se o protagonismo do serviço de saúde no acesso à biotecnologia, instituindo uma nova racionalidade de prevenção para os usuários em que a medicação é central em detrimento da com- 
preensão e busca de ação integral sobre as questões sociais e programáticas. Presume-se uma reação em cadeia em que uma tecnologia biomédica "puxa" a outra num cotidiano de ofertas centradas nos indivíduos. Assim, a busca pelo TR facilmente legitimava a oferta da PEP, pois compartilham a lógica biomédica ${ }^{4}$. Conjuntamente, tais tecnologias, operadas pela interlocução com as experiências pessoais de risco e doença das pessoas, implicam efeitos biopolíticos pelos quais se busca controlar a vida das populações 23 .

Mesmo como serviço especializado, o SAE deve ser parte de uma rede articulada de ações e serviços, visando à integralidade do cuidado. No entanto, verificou-se que a orientação programática da política de ampliar a oferta PEP gera influxos contrários a tal objetivo. Sobre isso, Das \& Poole 24, ao tratarem de temas que estão nas margens ou nas fronteiras, como migração, raça e xenofobia, teorizam sobre as relações entre centro e periferia. O Estado constrói protocolos, diretrizes e políticas para dirigir a vida dos sujeitos e, contudo, o próprio Estado teria suas margens espaciais e sociais reescritas pela forma como elas são traduzidas pelos serviços e profissionais considerando seus cenários locais.

Dados recentes da Coordenação Estadual de IST/AIDS mostram um aumento vertiginoso no uso da PEP no Rio Grande do Sul. Em 2007, apenas 481 PEP foram reportadas. Já em 2017, no escopo da Cooperação Interfederativa, foram realizados 2.932 procedimentos de PEP, representando um aumento de mais de seis vezes. Destaca-se que, em 2007, existia apenas o protocolo da PEP Ocupacional e Violência, sendo que em 2010, com o protocolo da PEP Sexual, iniciou um aumento tímido da procura. Por fim, em 2017, foi lançado o novo protocolo que ampliava a aplicação da PEP.

Quanto ao gênero, prática sexual e tipo de exposição dos/as usuários/as da PEP em 2017, o SAE acessado possuía as informações detalhadas na Tabela 1. Inicialmente, sobressai a ausência da referência às pessoas transexuais, população-chave para essa política, ainda que a ficha de atendimento do serviço contemplasse essa questão. Segundo a coordenadora, foram tão poucas pessoas transexuais que utilizaram a PEP em 2017 que elas foram consideradas na categoria HSH, pois todas eram mulheres trans. De qualquer modo, grande parte do volume de dispensação da PEP continuava sendo para situações de exposição ocupacional tanto para homens quanto para mulheres. Informações sobre raça/cor e escolaridade dos/as usuários/as não eram levantadas rotineiramente pelo SAE, indicando a invisibilidade de aspectos diferenciais da vulnerabilidade ao HIV no cotidiano de implementação da PEP. Por fim, em que pese a possibilidade de que o sexo com homens possa não ser referido pelos usuários, destaca-se a baixa procura de HSH pela PEP, outra população prioritária para a política.

Esperava-se que a novidade trazida por estratégias como a PEP fosse objeto de divulgação tanto para a população geral como para as populações específicas. Contudo, não se observou isso no campo, ensejando que a ampliação do acesso a tecnologias preventivas biomédicas atingia apenas pretensamente uma dimensão programática de enfrentamento da epidemia e acabava reforçando a apropriação meramente individual da PEP, ou seja, daqueles indivíduos mais bem informados e em melhores condições de acessar seus direitos. Por exemplo, verificou-se que nos SAE, principais portas de entrada para a PEP definidas na política 4,7 , não existia qualquer material informativo sobre

\section{Tabela 1}

Perfil geral e tipo de exposição de usuários/as da profilaxia pré-exposição (PEP) no Serviço de Assistência Especializada do Rio Grande do Sul, Brasil, em 2017.

\begin{tabular}{lccc}
\hline Tipo de exposição & Homem & HSH & Mulher \\
& Heterossexual & 9 & 29 \\
Relação sexual desprotegida consentida & 30 & 4 & 6 \\
Parceria HIV+ & 2 & 0 & 3 \\
Violência sexual & & 34 & 37 \\
Ocupacional & & 79 & 75 \\
Total & & & 37 \\
\hline
\end{tabular}

$\mathrm{HSH}$ : homens que fazem sexo com homens. 
a PEP ou a prevenção combinada. Materiais como cartazes e fôlderes continham informações sobre o TR, transmissão vertical e outras IST, em uma abordagem heteronormativa voltada às mulheres heterossexuais em idade reprodutiva. Embora alguns profissionais tenham ratificado que a PEP era prioritária para as populações-chave, consoante a orientação nacional e estadual para implementação da estratégia 3,4, não se encontrou nenhum material direcionado a tais populações nos serviços acessados. Segundo a psicóloga do SAE, a divulgação para essas populações ocorria em parceria com uma Organização Não-Governamental (ONG), ensejando que estaria disponível nos contextos sociais mais próximos dessas pessoas, ainda que causasse estranheza não estar também visível e sendo utilizado pelos serviços.

Questiona-se como essas pessoas chegam realmente a usufruir da PEP diante das barreiras de acesso aos cuidados em saúde pela população LGBT, já solidamente evidenciadas na literatura 5. Coadunando com isso, a quase totalidade dos usuários observados acessando a PEP nos serviços visitados eram homens na faixa etária de 30 a 40 anos, aparentemente de classe média, indicando que o acesso se intersecciona com classe social, raça e também com hierarquias sexuais 25 , reproduzindo iniquidades em saúde. Também não foram vistos/as travestis e transexuais nas observações ou nos registros do serviço. Por outro lado, os relatos e ações dos profissionais de saúde evidenciavam discursos morais quanto a essas populações atrelados às categorias de risco, podendo configurar-se como mais uma barreira: "Ele fazia sexo com homens e mulheres, bi, uma conduta muito vulnerável, muito vulnerável. Também acabou contraindo o HIV e de uma conduta muito vulnerável, de prostituição também, mas com uma questão emocional bem difícil" (Psicóloga).

A primeira dificuldade de acesso das travestis, transexuais e profissionais do sexo constatada era o horário de atendimento $(8: 30 \mathrm{~h}$ às $17 \mathrm{~h})$ que dificultava que muitas dessas pessoas, que trabalham à noite, buscassem o serviço. Para a coordenadora, a dificuldade principal era que os demais serviços de portas de entrada para a PEP (atenção primária à saúde - APS e a RUE) não estariam preparados para fornecer o "pacote completo" da PEP com aconselhamento e acompanhamento. Novamente, percebese um empobrecimento da prevenção combinada, pois, mesmo que contemple o aconselhamento e acompanhamento, essas ações seguem, no geral centradas no indivíduo e no acesso ao componente biomédico, considerando, de modo limitado, os contextos sociais e programáticos que geram suscetibilidades diferenciais ao HIV.

A Coordenação Estadual de IST/AIDS do Rio Grande do Sul, em 2017, norteava suas ações inspiradas no processo da Mandala da Prevenção Combinada 3. A Mandala do Rio Grande do Sul é dividida em três eixos chaves: prevenção, assistência e especificidades. Esse símbolo, representação da política de IST/aids do Estado, sugere uma dinamicidade "em que os eixos e elos se movimentam e se intercomunicam" 3 (p. 15). Todavia, a pesquisa revelou que os eixos que compõem a Mandala se organizam em ações específicas de prevenção, demonstrando pouca integração. Por exemplo, o TR, ação mais difundida até aquele momento, era ofertado nos SAE no âmbito dos municípios, sendo a APS responsável pelo seu agendamento, com exceção de Porto Alegre, em que a testagem foi matriciada em 2012. Ainda, era possível realizar o TR nas campanhas Fique Sabendo, em eventos pontuais no Dia de Luta Contra Aids, dia dos namorados, no Carnaval ou em agendas locais de grandes eventos que não têm o perfil de atingir uma população específica. Como no TR, a construção do fluxo da PEP informava que ela era realizada nos SAE e na RUE. Contudo, nos municípios pesquisados, a emergência só realizava a PEP quando o SAE não estava funcionando. Caso um usuário procurasse a emergência em horário comercial, era encaminhado para o SAE, o que podia se tornar uma barreira de acesso, pois demandava deslocamento e tempo. Sobre realizar a PEP na RUE, a coordenadora do SAE relatou: "Eu acho assim, a gente não pode querer que a Rede de Urgência e Emergência façam esse tipo de trabalho. Isso deveria ser feito onde? Lá no Serviço Especializado, que seriam aqueles ditos CTA de antigamente, que eram centros de testagem, onde as pessoas iam pra se testar e que na época a única prevenção que se tinha era o uso do preservativo. Então eu entendo que esses lugares deveriam ser minimamente reativados, pra que pudessem fazer esse trabalho. Porque não adianta tu simplesmente ir na emergência e receber o medicamento e tomar ou não, porque a grande maioria a gente sabe que nem toma os 28 dias. Mas que ele pudesse ser atendido depois nesse lugar, que ele fosse lá sim, que nesse lugar uma equipe multidisciplinar sentasse com ele e trabalhasse a prevenção combinada, escolher pra ele qual o melhor método de prevenção para aquele indivíduo naquele momento não é 'medicalizar' a profilaxia do HIV, mas usá-la como (mais) uma das estratégias de prevenção". 
Na contramão do preconizado na linha de cuidado para PVHA ${ }^{3}$ do Rio Grande do Sul, que coloca a APS como a porta de entrada para a prevenção e assistência em IST/HIV/aids, percebeu-se uma centralização da PEP, assim como do TR, nos SAE, e os outros pontos da rede apenas encaminhavam e/ ou referendavam o serviço especializado. Nesse sentido, a coordenadora do SAE pontua a dificuldade do acompanhamento dos usuários e a falta de informação sobre o desfecho do atendimento: "Atualmente, o que está acontecendo, a gente tem essa rede, mas de que maneira as pessoas estão sendo atendidas, se elas estão sendo atendidas no tempo que deve ser, se elas estão sendo bem acolhidas, a gente não tem esse dado não. A gente pode pelos dados logísticos ver que realmente, de 2015 para cá, aumentou o número de pessoas que retiraram medicamentos pra PEP sexual, mas quem eram essas pessoas e se elas concluíram o tratamento, nós não temos esse dado".

Pelas fragilidades da rede e da implementação da prevenção combinada, outra profissional chega a questionar a aplicação de recursos para a PEP quando faltavam ARV para quem já era infectado pelo HIV. Nesse caso, a cidadania biológica, possibilitada pelo acesso à PEP, é posta em cheque mediante uma posição que polariza os que "lutam pela vida", mais dignos de receber a medicação, contra os que "querem arriscá-la" sem usar preservativo. Aqui, percebe-se a operação de uma economia moral, em que o acesso à PEP é considerado um privilégio para pessoas consideradas moralmente menos merecedoras (porque não "querem" se cuidar) e que podem prejudicar aquelas que efetivamente já estão doentes: "No Brasil, está faltando medicamentos para PVHA, então como podemos oferecer para PEP? Qual é a prioridade? Possibilitar que os sujeitos vivam bem ou possibilitar que as pessoas deixem o preservativo de lado?" (Enfermeira).

Por fim, acrescenta-se que, diferentemente da normativa do Ministério da Saúde, que "recomenda a reavaliação da pessoa exposta após 2 semanas de PEP” 7 (p. 38) para identificar possíveis efeitos adversos e reforçar a necessidade da adesão até o final da quarta semana, constatou-se que isso não estava acontecendo pela falta de articulação entre os serviços da rede. Portanto, o fluxo da PEP nos documentos oficiais obedece às diretrizes da política nacional 4 , mas quando descemos para as margens, nos serviços, constata-se que essa tradução é feitas de várias maneiras, tendo em conta as limitações e fragilidades dos contextos assistenciais. Rasuras também aparecem nessa tradução, representadas pelo acesso desigual entre as populações-chave e por lógicas moralizantes.

\section{Considerações finais}

O trabalho buscou problematizar a complexa relação entre biomedicina, Estado e serviços, evidenciando como ela se tensiona num momento em que se apresentam novas "balas mágicas". Concomitantemente, sublinhou-se um forte potencial para que algumas vidas sigam sendo menos merecedoras de atenção. Assim, na intersecção entre globalização farmacêutica, políticas de saúde, gestão, assistência e usuários, a cidadania, mesmo restrita ao âmbito biológico, era incitada para alguns e restringida para outros, limitando-se o direito à prevenção, ao acesso e ao consumo individual da PEP.

Neste artigo, examinou-se como a economia política dos produtos farmacêuticos, que inspira políticas de tratamento globais e nacionais, se articula com mudanças institucionais e médicas que ocorreram no Brasil e no campo da aids nos últimos anos. Como um subproduto da ampliação do tratamento da aids, um modelo centrado no ponto de vista farmacêutico surgiu em meio ao desmantelamento de políticas sociais e de iniciativas de base comunitária para o tratamento e assistência de qualidade em saúde 26. Embora a mortalidade por aids tenha declinado 1,2, a ênfase no acesso à medicação também parece promover modelos que excluem alguns segmentos da população.

Pesquisas sobre as estratégias biomédicas têm sido valorizadas e são prioritárias nas gestões de saúde 9 . Não se trata de negar as possibilidades que a PEP oferece, mas de entender como a implementação dessa estratégia pode reproduzir e aprofundar iniquidades em saúde, tendo por base as fragilidades programáticas do SUS. Somando-se a isso um cenário de ataques conservadores às políticas sociais, tornando-se, portanto, importante mobilizar esforços para proteger a conquista que o acesso à PEP representa, aprimorando e complexando as ofertas preventivas que visam à integralidade e à equidade, sob pena de ser liquidada pela lógica do cálculo econômico.

Contudo, como se viu, há dificuldades quando se chega aos serviços, sobretudo na relação dos profissionais de saúde com os usuários e as outras estratégias de prevenção que compõem a Mandala 
do Rio Grande do Sul. Perceberam-se diversas formas pelas quais os profissionais e serviços de saúde (re)interpretam a PEP, resultando em uma oferta individualizante e descontextualizada, limitada do ponto de vista do direito à prevenção, da equidade e dos determinantes estruturais. Como outros pesquisadores nos lembram 13,14, é fundamental a política de aids revisitar sua resposta inicial quando o protagonismo dos movimentos sociais e a perspectiva ampliada dos direitos humanos davam o tom de ações preventivas que tiveram respostas potentes. Assim, o cardápio disponível das estratégias preventivas poderá dialogar efetivamente com os contextos sociais e práticas sexuais dos usuários, permitindo construir uma política universal e descentralizada que respeite a diversidade e priorize os mais vulneráveis.

No contexto da prevenção combinada, será útil que futuros estudos investiguem como essas novas tecnologias preventivas (PEP e PrEP) têm sido interpretadas e significadas pelos usuários, que uso eles têm feito delas e como esses diferentes usos se desenvolvem no contato com os serviços, considerando as desigualdades sociais. A medicalização favorece uma perspectiva individual 27 , diferente de pensar a prevenção pela perspectiva dos direitos humanos. O desafio que se apresenta aos profissionais e gestores de saúde é pensar ações que transcendam a oferta do medicamento, sob pena de afastar-se dos fundamentos preconizados pela própria política e distante do ideal resgatado na fala da profissional: "não é 'medicalizar' a profilaxia do HIV, mas usá-la como uma das estratégias de prevenção" (Coordenadora do SAE).

\section{Colaboradores}

A. H. C. Costa concebeu o estudo, realizou o trabalho de campo e as análises, confeccionou a primeira versão do artigo e aprovou a versão final. T. R. Gonçalves concebeu o estudo, colaborou nas análises, revisou criticamente o texto e aprovou a versão final.

\section{Conflito de interesses}

A. H. C. Costa foi consultor da Coordenação Estadual de IST/AIDS do Rio Grande do Sul de 2013 a 2018.

\section{Informações adicionais}

ORCID: Adriano Henrique Caetano Costa (00000002-1207-5320); Tonantzin Ribeiro Gonçalves (0000-0003-0249-3358).

\section{Agradecimentos}

Agradecemos especialmente a todos/as interlocutores/as da pesquisa ligados aos serviços da rede de atenção em HIV/aids acessados. À Coordenação de Aperfeiçoamento de Pessoal de Nível Superior (CAPES; Código de Financiamento 001). 


\section{Referências}

1. Departamento de Vigilância, Prevenção e Controle das IST, HIV/AIDS e Hepatites Virais, Secretaria de Vigilância em Saúde, Ministério da Saúde. Boletim Epidemiológico - Aids e IST 2016/2017; Ano V, no 1 - 27a a 53a semanas epidemiológicas - julho a dezembro de 2016; no 1 - 1a a 26a - semanas epidemiológicas - janeiro a junho de 2017.

2. Batista CST, Oliveira TH. Boletim epidemiológico: HIV/aids. Porto Alegre: Secretaria de Estado da Saúde do Rio Grande do Sul; 2018.

3. Secretaria de Estado da Saúde do Rio Grande do Sul. Linha de cuidado para pessoas vivendo com HIV/aids (PVHA) e outras DST. Porto Alegre: Secretária Estadual de Saúde do Rio Grande do Sul; 2014.

4. Departamento de Vigilância, Prevenção e Controle das IST, HIV/AIDS e Hepatites Virais, Secretaria de Vigilância em Saúde, Ministério da Saúde. Prevenção combinada do HIV: bases conceituais para profissionais, trabalhadores(as) e gestores(as) de saúde. Brasília: Ministério da Saúde; 2017.

5. Ayres JR, Paiva V, Buchalla C. Direitos humanos e vulnerabilidade na prevenção e promoção da saúde: uma introdução. In: Paiva V, Ayres JR, Buchalla C, organizadores. Vulnerabilidade e direitos humanos: prevenção e promoção da saúde - Livro I. Curitiba: Juruá; 2012. p. 9-22.

6. Mann J, Tarantola DJM, Netter TW. A aids no mundo. Rio de Janeiro: Relume-Dumará; 1993.

7. Departamento de Vigilância, Prevenção e Controle das IST, HIV/AIDS e Hepatites Virais, Secretaria de Vigilância em Saúde, Ministério da Saúde. Protocolo clínico e diretrizes terapêuticas para profilaxia pós-exposição (PEP) de risco à infecção pelo HIV, IST e hepatites virais. Brasília: Ministério da Saúde; 2017.

8. Grangeiro A, Ferraz D, Calazans G, Zucchi EM, Díaz-Bermúdez XP. The effect of prevention methods on reducing sexual risk for HIV and their potential impact on a large-scale: a literature review. Rev Bras Epidemiol 2015; 18 Suppl 1:43-62.

9. Kuchenbecker, R. What is the benefit of the biomedical and behavioral interventions in preventing HIV transmission? Rev Bras Epidemiol 2015; 18 Suppl 1:26-42.

10. Biehl J, Petryna A. Tratamentos jurídicos: os mercados terapêuticos e a judicialização do direito à saúde. Hist Ciênc Saúde-Manguinhos 2016; 23:173-92.

11. Rose N, Novas C. Biological citizenship. In: Ong A, Collier S, editors. Blackwell companion to global anthropology. Oxford: Blackwell; 2003. p. 439-63.
12. Arendt H. A condição humana. 10a Ed. Rio de Janeiro: Editora Forense Universitária; 2000.

13. Grangeiro A, Castanheira E, Nemes MI. A re-emergência da epidemia de aids no Brasil: desafios e perspectivas para o seu enfrentamento. Interface Comum Saúde Educ 2016; 19:5-8.

14. Seffner F, Parker R. Desperdício da experiência e precarização da vida: momento político contemporâneo da resposta brasileira à aids. Interface Comum Saúde Educ 2016; 20:293304.

15. Knauth D. A etnografia na saúde coletiva: desafios e perspectivas. In: Schuch P, Steffen M, Peters R, organizadores. Experiências, dilemas e desafios do fazer etnográfico contemporâneo. Porto Alegre: Editora da UFRGS; 2010. p. 109-14.

16. Geertz C. O saber local. Petrópolis: Editora Vozes; 2000

17. Marcus GE. A estética contemporânea do trabalho de campo na arte e na antropologia: experiências em colaboração e intervenção. In: Barbosa A, Cunha ET, Hikiji RS, organizadores. Imagem-conhecimento: antropologia, cinema e outros diálogos. Campinas: Papirus; 2009. p. 13-32.

18. Biehl J, Amon JJ, Socal MP, Petryna A. Between the court and the clinic: lawsuits for medicines and the right to health in Brazil. Health Hum Rights 2012; 14:E36-52.

19. Rose N. A política da própria vida: biomedicina, poder e subjetividade no século XXI. São Paulo: Paulus; 2013.

20. Carrara, S. Tributo a Vênus. A luta contra a sífilis no Brasil: da passagem do século aos anos 40. Rio de Janeiro: Editora Fiocruz; 1996.

21. Gibbon S, Novas C. Introduction: biosocialities, genetics and the social sciences. In: Gibbon S, Novas C, editors. Biosocialities, genetics and the social sciences: making biologies and identities. London: Routledge; 2008. p. 1-17.

22. Santos RV, Maio MC. Race, genomics, identities and politics in contemporary Brazil. Crit Anthropol 2004; 24:347-78.

23. Rabinow P, Rose N. Biopower today. Biosocieties 2006; 1:195-217.

24. Das V, Poole D. Anthropology in the margins of the state. Santa Fe: School of American Research Press; 2004.

25. Rubin G. Thinking sex: notes for a radical theory of the politics of sexuality. In: Rubin G, editor. Culture, society and sexuality: a reader. London: UCL Press; 1999. p. 143-78.

26. Paim J. Sistema Único de Saúde (SUS) aos 30 anos. Ciênc Saúde Colet 2018; 23:1723-8.

27. Maksud I, Fernandes N, Filgueiras S. Technologies for HIV prevention and care: challenges for health services. Rev Bras Epidemiol 2015; 18:104-19. 


\section{Abstract}

Based on a multicenter ethnographic field survey on the implementation of post-exposure prophylaxis (PEP) in the State of Rio Grande do Sul, Bra$z i l$, this article discusses aspects of micropolitics in the supply of "new preventive technologies" based on the concepts of pharmaceutical globalization and biological citizenship. Combined prevention suggests the joint use of behavioral, biomedical, and structural strategies to confront HIV, but on the margins of this policy's co-construction, actions by health services in Rio Grande do Sul showed a profound fragmentation of this combination in the network, prioritizing access to medication. Stimulated by a scenario of pharmaceutical globalization, the way PEP is accessed and supplied to users encourages a biological citizenship that involves the right to prevention, in this case through the individual right to consume the medication. However, access to this right is permeated by moral conditioning factors linked to risk categories and by the users' social context, reproducing inequalities in health and impoverishing the counseling approach. The article discusses the need for combined prevention strategies that strengthen the approach of social and program dimensions in the epidemic, which will also bolster the attention to individual vulnerabilities from the perspective of comprehensive health.

HIV; Acquired Immunodeficiency Syndome; Post-exposure Prophylaxis; Health Vulnerability

\section{Resumen}

Basándose en una investigación etnográfica multisituada en el campo de la implementación de la profilaxia posexposición (PEP) en servicios de salud del Estado de Río Grande do Sul, Brasil, este estudio discute aspectos de la micropolitica de oferta de las "nuevas tecnologías de prevención", a partir de los conceptos de globalización farmacéutica y ciudadanía biológica. La prevención combinada sugiere el empleo conjunto de estrategias comportamentales, biomédicas y estructurales para enfrentar el VIH, sin embargo, en los márgenes (co)construidos de esa politica, las acciones de los servicios de salud observados en Rio Grande do Sul evidenciaron la profunda fragmentación de esta combinación en red, privilegiándose el acceso a la medicación. Estimulada por un escenario de globalización farmacéutica, la forma como se accede a la PEP y se ofrece a los usuarios da la oportunidad a la creación de una ciudadanía biológica que implica el derecho a la prevención, en este caso mediante el derecho individual a consumir la medicación. No obstante, el acceso a tal derecho es traspasado por condicionamientos morales, vinculados a las categorías de riesgo y por el contexto social de los usuarios, reproduciéndose inequidades en salud y empobreciendo el planteamiento de asesoramiento. Se discute la necesidad sobre qué estrategias de prevención combinada fortalecen el planteamiento de las dimensiones sociales y programáticas de la epidemia, lo que también potencializará la atención a las vulnerabilidades individuales desde la perspectiva de la salud integral.

VIH; Síndrome de Inmunodeficiencia Adquirida; Profilaxis Posexposición; Vulnerabilidad en Salud

Recebido em 04/Mar/2020

Versão final reapresentada em 18/Mai/2020

Aprovado em 19/Jun/2020 